\section{LIMITACIONES DEL ANÁLISIS SECUNDARIO DE BASES DE DATOS - RÉPLICA}

\section{LIMITATIONS OF SECONDARY ANALYSIS OF DATABASES - REPLY}

\author{
Oscar Munares-García ${ }^{1,2, a}$, \\ Guillermo Gómez-Guizado ${ }^{1, b}$, \\ Juan Barboza-Del Carpio ${ }^{1, c}$, \\ José Sánchez-Abanto ${ }^{1, d}$
}

Sr. Editor. Permítanos responder a los comentarios realizados a nuestro artículo:

- La denominación de "estudio transversal" forma parte de una amplia clasificación del tipo de estudio ${ }^{(1)}$. La secuencia transversal se refiere a que las variables fueron medidas una sola vez, que es lo que se ha descrito en el artículo (2).

- Las investigaciones cuantitativas deben evitar el error sistemático y aleatorio ${ }^{(3)}$, el análisis de información secundaria no escapa de estos puntos. El sistema SIEN cuenta con un sistema de corrección de errores que está adecuadamente descrito en la sección "Materiales y métodos". Los comentarios emplean el artículo de Ayala et al. ${ }^{(4)}$, que indica literalmente que: El sistema estadístico nacional debería aprovechar la gran cantidad de información disponible en la administración pública /.../, mencionando como ventajas que (1) si la información ya existe no es necesario volver a obtener la misma información con fines de estudio o investigación; (2) el ahorro de costos es considerable; y (3) la riqueza informativa contenida representa un activo de conocimiento de la realidad susceptible de ser aprovechado en beneficio de la ciudadanía. También mencionan que dichos registros deben ser evaluados con rigor si se trata de usarlo como fuente estadística, especificando la depuración y el control de calidad. Sin embargo, Ayala et al. se explaya en los enlaces de varias fuentes de datos donde menciona: La

\footnotetext{
1 Centro Nacional de Alimentación y Nutrición, Instituto Nacional de Salud. Lima, Perú.

2 Departamento Académico de Obstetricia, Facultad de Medicina Humana, "San Fernando" Universidad Nacional Mayor de San Marcos. Lima, Perú. a Licenciado en Obstetricia magister en Salud Pública; ${ }^{b}$ médico cirujano especialista en Epidemiologia de Campo; ${ }^{c}$ nutricionista; ${ }^{d}$ nutricionista magister en Nutrición

Recibido: 05-02-13 Aprobado: 06-02-13
}

Citar como: Munares-García O, Gómez-Guizado G, Barboza-Del Carpio J, Sánchez-Abanto J. Limitaciones del análisis secundario de bases de datos Réplica [carta]. Rev Peru Med Exp Salud Publica. 2013;30(1):152-3. posibilidad de sesgos de un sistema de información en una base longitudinal de microdatos válida para la investigación de políticas sociales y la evaluación de programas sociales. Esta situación no se ajusta a lo realizado, ya que hemos usado solo un tipo de registro estandarizado a nivel nacional, el cual incluso no es sofisticado, tal como lo recomienda el artículo de Hotz et al. ${ }^{(5)}$.

- A partir del segundo trimestre del embarazo se produce la denominada hiperhidrosis gestacional, con disminución de los niveles de hemoglobina, razón por la cual se ha estimado los niveles de hemoglobina por trimestre. En situaciones ideales esta primera medición ocurre en el primer trimestre del embarazo, ello ocurre en el $74,9 \%$ de las gestantes (ENDES 2011), con la finalidad que para el segundo trimestre se realice un nuevo dosaje y se compruebe la corrección de la anemia; consideramos ventajoso tomar el último dosaje, pues permitirá determinar la prevalencia de anemia en el último contacto con el establecimiento de salud, independientemente a la administración de tratamiento.

- El cálculo de la prevalencia está en función del denominador, el "problema del denominador", mencionado en el artículo de Hotz et al., no ha sido problema en nuestro estudio, ya que en nuestro artículo se menciona que el denominador son las gestantes que acuden a los establecimientos de salud del Ministerio de Salud.

- La determinación de estimaciones se produce tanto en poblaciones o en muestras, la variable hemoglobina correspondió al $54,7 \%$ de la población estimada de gestantes para el Ministerio de Salud, por lo que consideramos apropiado determinar si existían homogeneidad de las varianzas encontradas, los intervalos de confianza fueron sugeridos por los pares revisores.

- La determinación de la hemoglobina en el primer control, o el último, no cumple un papel fundamental en la determinación de una prevalencia, puesto que cuando se hacen estudios poblacionales esta variable también se presenta y no interfiere en la determinación de una prevalencia de periodo. Sin embargo, en la comparación con el estudio de Gonzales sí podría ser pertinente, ya que la primera medición se realiza usualmente en el primer trimestre de gestación (estudio basado en un análisis secundario de datos del Sistema Informático Perinatal -SIP 2000-, similar al nuestro), en cuyo caso sus resultados serían muy cercanos a lo encontrado en nuestro artículo en el primer 
trimestre de gestación, a pesar de que dicho autor no realiza el ajuste por la altitud.

Esperamos que estas aclaraciones sirvan para comprender mejor nuestro artículo.

\section{REFERENCIAS BIBLIOGRÁFICAS}

1. Pineda EB, de Alvarado EL. Metodologia de la Investigación. 3ra ed. Washington: OPS ; 2008.

2. Munares-García O, Gómez-Guizado G, Barboza-Del Carpio J, Sánchez-Abanto J. Niveles de hemoglobina en gestantes atendidas en establecimientos del Ministerio de Salud del Perú, 2011. Rev Peru Med Exp Salud Publica. 2012;29(3):329-36.

3. Rothman KJ. Epidemiología moderna. Madris: Ediciones Diaz Santos; 1987.

4. Ayala Cañón L, Rodríguez Coma M. La utilización de registros administrativos como base para la investigación de políticas sociales. Seminario Internacional: El papel de los registros administrativos en relación con el análisis social y económico y el desarrollo del Sistema Estadístico Nacional. Instituto de Estudios Fiscales, Madrid, Esapaña, 30 y 31 de enero de 2006.

5. Hotz VJ, Goerge R, Balzekas J, Margolin F (Editores). Administrative data for policy-relevant research: assessment of current utility and recommendations for development. A Report of the Advisory Panel on Research Uses of Administrative Data. Illinois: Northwestern University/University of Chicago/Joint Center for Poverty Research; 1998.

Correspondencia: Oscar Fausto Munares García

Dirección: Av. Tizón y Bueno 276. Lima 11, Perú.

Teléfono: (511) $7480060 / 998719537$

Correo electrónico: omunares@ins.gob.pe
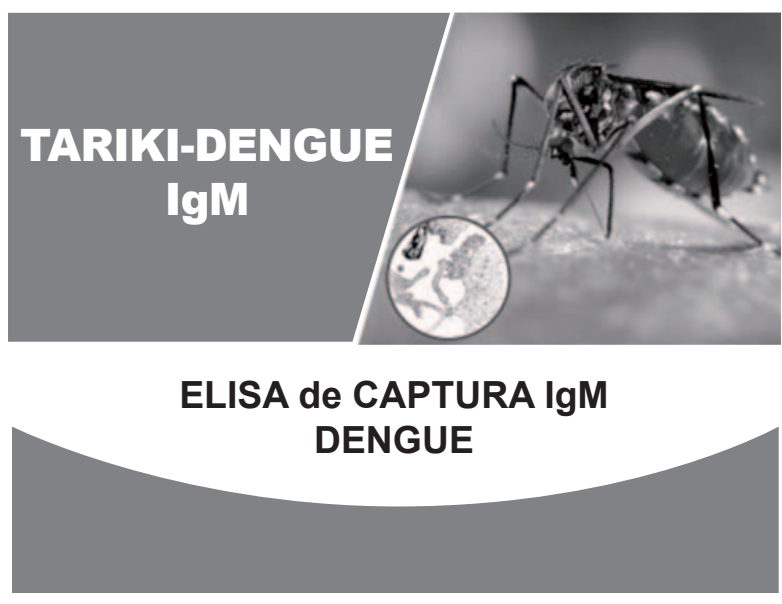

TARIKI: disponible en el Perú

Kit para la determinación de anticuerpos IgM anti Dengue, desarrollado y producido por el Instituto Nacional de Salud, Perú INS/MINSA

\section{CRECIMIENTO FETAL Y PESO AL NACER EN LA ALTURA}

\author{
FETAL GROWTH AND BIRTH WEIGHT \\ IN THE HIGHLANDS
}

\author{
Wilfredo Villamonte ${ }^{1,2, a}$, María Jerí 1,b
}

Sr Editor. A propósito del artículo "Impacto de la altura en el embarazo y el producto de la gestación" (1), queremos comentar que el peso al nacer en la altura se ha definido que es menor al correspondiente al nivel del mar, y no es una restricción de crecimiento, ya que no existe una redistribución de flujo hacia el cerebro fetal (2).

El peso al nacer disminuye conforme se asciende en altura en el Perú (3), y consideramos que la pobreza sí tiene un efecto independientemente de la altura en el crecimiento fetal, es por eso que los neonatos provenientes de los hospitales de EsSalud, tienen pesos mayores a los correspondientes del Ministerio de Salud (MINSA) independientemente de la altura. Un ejemplo de ello es el peso promedio que hallamos en Lima para EsSalud, que es $3383 \mathrm{~g}$ y para los $3400 \mathrm{~m}$ de altura de $3262 \mathrm{~g}$. Estos valores son mayores al peso al nacer descrito para los hospitales del MINSA, en Lima, donde el peso promedio al nacer es $3260 \mathrm{~g}$ y a $3400 \mathrm{~m}$ de altura es $3090 \mathrm{~g}$.

La población de EsSalud del Cusco muestra mejores características socioeconómicas (4) que las correspondientes gestantes que acuden a los hospitales del MINSA, un ejemplo de ello es que los padres tienen una educación superior en $85 \%$, al igual que $79 \%$ de las madres, pero los primeros en un $75 \%$ se desempeñan como profesionales mientras que las segundas llegan a $47 \%$. El $99 \%$ de estas parejas tienen una unión estable (casados o convivientes) y poseen un ingreso económico mayor a 1000 soles mensuales en un $72 \%$. La tasa de mortalidad materna en los últimos años ha sido de cero en este hospital.

El crecimiento fetal evaluado por ultrasonografía tiene algunas particularidades. La circunferencia de la cabeza y abdomen al igual que la longitud del fémur a 3400 m

\footnotetext{
Departamento de Ginecobstetricia, Hospital Adolfo Guevara Velazco, EsSalud. Cusco, Perú.

2 Universidad Nacional de San Antonio Abad del Cusco. Cusco, Perú.

Médico ginecobstetra, ${ }^{\mathrm{b}}$ obstetriz

Recibido: 12-02-13 Aprobado: 20-02-13
}

Citar como: Villamonte W, Jerí M. Crecimiento fetal y peso al nacer en la altura [carta]. Rev Peru Med Exp Salud Publica. 2013;30(1):153-4. 